\title{
Optimization of the Operation of Hydro Stations in Market Environment Using Genetic Algorithms
}

\author{
G. S. Sampaio, J. T. Saraiva, Member IEEE, J. C. Sousa and V. T. Mendes
}

\begin{abstract}
This paper describes an approach to the short term operation planning of hydro stations in market environment. The developed approach is based on the solution of an optimization problem to maximize the profit of a generation agent along a planning period discretized in hourly steps using a Genetic Algorithm. This problem includes the possibility of pumping since this is an important resource in the scope of electricity markets. The scheduling problem was developed starting with an initial simplified version in which the head loss is neglected and the head is assumed constant. Then, it was implemented a second model in which the nonlinear relation between the head, the hydro power and the water discharge is retained and finally an approach in which the hydro schedule obtained in a given step is used to update the hourly electricity prices used to compute the profit of the generation agent. The short term hydro scheduling problem is illustrated using two Case Studies - the first one was designed to run a set of initial tests to the developed algorithm and the second one refers to a set of hydro stations that mirrors a cascade of 8 stations in Portugal.
\end{abstract}

Key words - short-term hydro scheduling, pumping, day-ahead market, profit maximization, genetic algorithms.

\section{INTRODUCTION}

Before the introduction of market mechanisms in the electricity sector, the industry was structured in a vertically way in terms of utilities that integrated all the activities of the value chain from generation till the relation with end consumers. Under this vertical paradigm, planning activities, both in terms of expansion and operation, were conducted in a centralized way. In particular for operation planning activities, traditional utilities conducted planning exercises using estimates of fuel and coal costs in order to obtain estimates of the contribution of each technology to supply the expected demand. Then, as the operation moment was approaching, more detailed studies were conducted including at a certain moment detailed network models that ultimately lead to the OPF problem.

In several countries the generation mix includes a large share of hydro stations so that a different set of concerns emerged related with the definition of strategies to use the water. It is clear that considering in the same optimization problem resources having non zero operation cost as

The first author thanks EDP Produção, for all the support and guidance during the preparation of his MSc thesis.

Gil Sampaio (gil.sampaio@fe.up.pt) completed his MSc at FEUP, Faculdade de Engenharia da Universidade do Porto in July 2012.

João Tomé Saraiva (jsaraiva@fe.up.pt)is with FEUP, Faculdade de Engenharia da Universidade do Porto and INESC Porto - Instituto de Engenharia de Sistemas e Computadores do Porto, Campus da FEUP, Rua Dr. Roberto Frias, 4200-465 Porto, Portugal.

José Carlos Sousa (jose.sousa@edp.pt) and Virgílio T. Mendes (virgilio.mendes@edp.pt) are with EDP Produção SA, Rua Ofélia Diogo da Costa, 45, 4149 - 022 Porto, Portugal. thermal stations together with hydro stations would lead to a more intensive use of hydro resources until water was available meaning that later on thermal stations would have to be used more intensively. This short term intensive use of water wasn't eventually the most interesting operation strategy and this concern was reflected on a number of studies that for instance originated the definition and the estimation of the value of water. These research activities originated a large number of publications reporting models on hydro-thermal coordination problems in which it was recognized the random nature of water inflows and the different characteristics of hydro stations, namely in terms of reservoirs and run of river stations.

More recently, the advent of market mechanisms introduced significant changes in this centralized vision. There are now several generation agents competing to supply the demand and each of them aiming at maximizing its own profit. The relation between generation and demand can now be done in symmetrical day-ahead markets to which generation and demand agents bid for each hour of the next day. Ultimately, the introduction of this type of procedures put a larger emphasis on shorter term concerns meaning that hydro selling bids are often formulated in terms of the maximum possible power to be generated namely when it is expected that electricity market prices are larger thus yielding larger profits. In this context, pumping becomes an important resource not only because if used efficiently it can contribute to increase the profits but also because it can be used to accommodate the energy from other volatile sources as wind, namely in windy valley periods.

The operation planning problem of hydro stations corresponds to a complex problem, namely because it corresponds to a non linear optimization problem given the non-linear relation between the head, the power output and the water flow and also because the number of constraints and variables becomes typically large if one aims at running a planning exercise for a realistic system. When addressing this short term operation problem it is important to recognize that it is necessary to specify values for instance associated with the initial and final volumes of water in each reservoir. These values typically come from medium or longer term planning exercises that use estimates of water inflows to each station and that outputs the initial and final volumes to use in each planning period, namely as a way to reduce or bring to zero water spill given that this corresponds to waste a valuable resource.

Having in mind these ideas, this paper formulates the short term hydro station operation planning problem that should be solved by a generation agent in order to obtain a 
generation schedule along a day or a week so that the corresponding profit is maximized while enforcing a number of constraints. When formulating this problem we admit that the initial and final water volumes of each station are specified and that it is also available a set of estimated hourly electricity prices. The short term hydro scheduling problem can then be used by a generation agent to formulate the selling bids to submit to the Market Operator which means that this schedule will then have an impact on the hourly prices. The paper also includes results obtained for several tests starting at a simplified version of the problem in which the head loss is neglected. Then, the non-linear relation between the head, the water flow and the power output is considered and finally the schedule obtained in a given step of the iterative solution algorithm is used to update the electricity market prices to calculate the profit of the hydro stations being addressed.

Accordingly, this paper is structured as follows. After this introductory section, Section II reviews several concepts related with the operation of hydro systems and briefly addresses a number of publications on the hydro scheduling problem and Section III presents the formulation of the short term problem. Then, Section IV details the application of GA to this problem. Section V presents results from two Case Studies designed to illustrate the developed approach and finally Section VI draws the most relevant conclusions.

\section{STATE OF THE ART}

As explained before, under the current competitive market paradigm, generation companies should identify the most interesting operation strategy for the next day or week. This identification should be driven by the maximization of their profits and the results of the corresponding short term planning exercises can then be used to build a set of selling bids to be communicated to the day-ahead market operator.

When considering generation companies having hydro assets the problem becomes more complex, namely due to its non linear nature together with the number of constraints and variables to consider and the interdependencies between several stations in cascades. In the first place, for an ideal hydro circuit associated with a station $\mathrm{i}$ and a period $\mathrm{k}$, the output power is given by (1). In this expression, the gravity acceleration is $9,8 \mathrm{~ms}^{-2}$ the density of the water is $1000 \mathrm{~kg} / \mathrm{m}^{3}, q_{i k}$ is the water flow in $\mathrm{m}^{3} \mathrm{~s}^{-1}, h_{i k}$ is the water head in $\mathrm{m}$ and $\mu_{T}$ is the generation efficiency factor.

$$
p_{i k}=9,8.1000 \cdot q_{i k} \cdot h_{i k} \cdot \mu_{T}
$$

In fact, the water head depends under a non-linear way on the water flow originating losses in the water circuit. Therefore, $p_{i k}$ will more accurately be represented by (2) where $\beta$ is the head loss coefficient. According to (2), for each value of $h_{i k}$, there is also a non-linear relation between $q_{i k}$ and $p_{i k}$. This family of curves is illustrated in Fig. 1, each of them for a fixed value of the head.

$$
p_{i k}=9,8.1000 \cdot q_{i k} \cdot\left(h_{i k}-\beta \cdot q_{i k}^{2}\right) \mu_{T}
$$

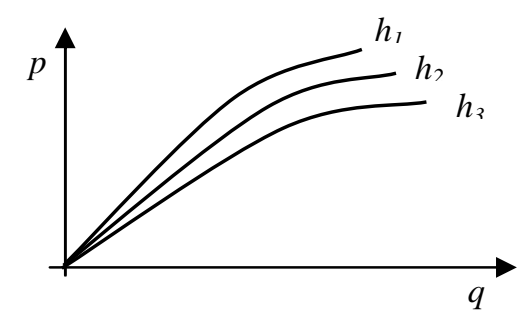

Fig. 1. Family of curves for the power output of an hydro station.

The head loss coefficient $\beta$ is given by (3) in which $\Delta h n$ is the nominal head loss and $q n$ is the nominal discharge flow. Once the $\beta$ is calculated, the head loss can be obtained by $\beta . q_{i k}^{2}$. Due to this term and even if a constant head $h_{i k}$ is set, the power output of the hydro station is still a non-linear function of the discharge flow as it can be recognized in each curve of Fig. 1.

$$
\beta=\frac{\Delta h n}{q n^{2}}
$$

The literature describes different strategies to deal with the head and discharge volume non-linear relation. In the first place, the problem could be simplified neglecting the head loss term in (2). Under this assumption, for each value of $h_{i k}$, the non-linear curve would be approximated by a linear relation between the hydro power output and the discharge flow. Given that the term $\beta . q_{i k}^{2}$ is neglected, this linear approximation gets located above the real curve in blue in Fig 2. Accordingly, this would lead to larger errors in the area of large discharge flows were the current practice indicates that hydro stations operate nowadays.

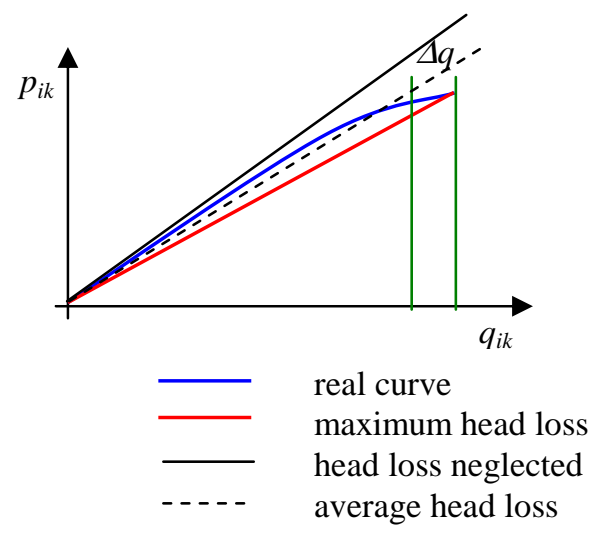

Fig. 2. Impact of the head loss in the hydro power output.

A second possibility represented by the dashed line in Fig. 2 corresponds to use an average value for the head loss that could be obtained by minimizing the average square error of the difference of values obtained from the blue curve and its dashed linear approximation. A third approach is a more conservative one in the sense that it uses a constant value for the head loss corresponding to the maximum discharge flow. In this case, for a given value of the discharge flow, the hydro power output given by the red curve is below the real value given by the blue curve. However, this approximation is interesting since the error is small in the area of larger discharge flows and the current practice of hydro station operation indicates that 
that the discharge flows are typically at the maximum.

In several cases, one can admit that the head $h_{i k}$ is constant, namely for large reservoirs in which even if there are large discharge flows the head slightly changes. Differently, assuming a constant head is not suitable for smaller reservoirs because they will have large variation levels depending on the discharge flow thus leading to head variations. In these cases, the mentioned non-linear relation should be retained and addressed in the model to obtain more accurate results. Therefore, depending on the main characteristics of the hydro system under analysis, this non-linear relation can be neglected or not.

The mentioned non-linear problem can be addressed using dynamic programming models as in $[1,2,3]$. The use of this technique usually leads to large dimension optimization problems if we are dealing with realistic sized hydro generation systems. Other publications describe the use of other techniques as Lagrange relaxation, mixed integer linear programming [4], non-linear formulations [5] and meta-heuristics. In this class, there are publications using Simulated Annealing [6], Neural Networks [7, 8] and Tabu Search [9]. Several publications use an iterative procedure in which the head is updated in each iteration using the value obtained for $q_{i k}$ in the previous iteration and the $\beta$ coefficient calculated with nominal values (3). This approach was used in $[10,11,12]$ and also in [13] considering an under relaxation iterative scheme.

In this paper we describe the mathematical formulation to the short term hydro scheduling problem and three possible implementation approaches designed so that they assume an increasing complexity level. These approaches were developed in the scope of the preparation of the MSc Thesis of the first author [14]. In the first one we used a constant value both to the head $h_{i k}$ and to the head loss, this one corresponding to the maximum discharge flow. This means that the relation between the hydropower output and the discharge flow becomes linear and it is the same all along the solution algorithm. Then, the second model incorporates the mentioned non-linear relation under an iterative scheme. After obtaining a solution for $q_{i k}$ the head loss term $\beta . q_{i k}^{2}$ and the net head $h_{i k}-\beta \cdot q_{i k}^{2}$ are updated and used in the next iteration. The third implementation considers the impact of the hydro schedule on the electricity market prices. The hydro schedule obtained in one iteration is used to update the electricity prices and these are then used to calculate the amounts the remuneration of each station along the planning period.

Finally, the developed approach includes the possibility of pumping to increase the realism of the models. In case of pumping, the power consumed by station $\mathrm{i}$ in period $\mathrm{k}$ is given (4) in which $\mu_{P}$ is the pumping efficiency factor.

$$
p_{i k}=9,8.1000 \cdot q_{i k} \cdot\left(h_{i k}+\beta . q_{i k}^{2}\right) / \mu_{P}
$$

\section{COMPLETE MATHEMATICAL FORMULATION}

The main objective of the Hydro Scheduling Problem (HSP) can be stated as follows - find a feasible operation schedule for a set of hydro stations (including for instance cascades) that maximizes the profit while enforcing a number of constraints. One of the main difficulties when trying to solve this problem corresponds to the non-linear relationship between the discharge volume $q_{i k}$, the net head $h_{i k}$, and the hydropower $p_{i k}$ as represented in Fig. 1. In the implemented approach we considered a time scale of $\mathrm{K}$ hourly periods and we assumed that a set of $\mathrm{K}$ hourly electricity prices, $\pi_{k}$, are available. Then this problem can be formulated by $(5-13)$.

$\max \sum_{i=1}^{I} \sum_{k=1}^{K}\left(\pi_{k} \cdot P_{T i k}\right)-\left(\pi_{k} \cdot P_{P i k}\right)-\left(p s \cdot s_{i k}\right)$

subj.

$$
\begin{aligned}
& v_{i k}=v_{i(k-1)}+a_{i k}-q_{T i k}-s_{i k}+q_{P i k}+ \\
& \quad+\sum_{m \in M i}\left(q_{T m\left(k-\phi_{m}\right)}+s_{m\left(k-\lambda_{m}\right)}-q_{P m\left(k-\omega_{m}\right)}\right) \\
& \operatorname{vol}_{i}^{\text {lmin }} \leq q_{T i k}+s_{i k}-q_{P i k} \leq v_{\text {mol }}^{\text {max }} \\
& v_{i}^{\text {min }} \leq v_{i k} \leq v_{i}^{\text {max }} \\
& q T_{i}^{\text {min }} \leq q T_{i k} \leq q T_{i}^{\text {max }} \\
& q P_{i}^{\text {min }} \leq q P_{i k} \leq q P_{i}^{\text {max }} \\
& O \leq s_{i k} \leq \infty \\
& v_{i K}=\operatorname{vol}_{i K} \\
& i=1, \ldots, I ; k=1, \ldots, K ; m=1, \ldots, L
\end{aligned}
$$

In this formulation:

- $\quad I$ - number of reservoirs;

- $\quad i$ - index for a particular reservoir;

- $\quad K$ - number of scheduling hourly periods;

- $\quad k$-index for a particular hour;

- $\quad M i$ - set of upstream reservoirs directly connected with reservoir i;

- $\quad m$-index for a reservoir in set $M i$;

- $\quad L$ - number of stations in set $M i$;

- $\pi_{k}$ - electricity market price in hour k;

- $\quad P_{T_{i k}}$ - power output in reservoir i, hour $\mathrm{k}$;

- $\quad P_{P_{i k}}$ - pumping power in reservoir i, hour k;

- $\quad p s$ - penalty factor for spills;

- $\quad s_{i k}$ - spill of reservoir $\mathrm{i}$, in hour $\mathrm{k}$;

- $\quad v_{i k}$-volume of reservoir $\mathrm{i}$, in hour $\mathrm{k}$;

- $a_{i k}-$ inflow of reservoir $\mathrm{i}$, in hour $\mathrm{k}$;

- $q_{P i k}$ - pumping volume of reservoir i, in hour k;

- $q_{\text {Tik }}$ - discharge volume of reservoir $\mathrm{i}$, in hour $\mathrm{k}$;

- $\phi_{m}$ - delay of turbine discharge volumes;

- $\lambda_{m}$ - delay of spill volumes;

- $\omega_{m}$ - delay of pumping volumes;

- $\operatorname{vol}_{i}^{l \min }$, vol $_{i}^{l m a x}$ - minimum and maximum launch volumes of reservoir I for instance considering ecologic requirements;

- $\quad v_{i}^{\min }, v_{i}^{\max }$ - level volume limits of reservoir $\mathrm{i}$;

- $\quad q T_{i}^{\min }, q T_{i}^{\max }$ - turbine discharge limits for station $\mathrm{i}$;

- $\quad q P_{i}^{\min }, q P_{i}^{\max }$ - pumping volume limits for station $\mathrm{i}$;

- $\operatorname{vol}_{i K}$ - volume level of reservoir $\mathrm{i}$ in the last scheduling period, $\mathrm{K}$.

In this formulation, the objective function (5) aims at 
maximizing the profit of the generation company given that at each period $\mathrm{k}$ the power output of each station $\mathrm{i}, P_{T_{i k}}$, is sold at the market price $\pi_{k}$. If station i is pumping water it consumes electricity that is bought at price $\pi_{k}$ thus representing a reduction of the revenue. Water spills correspond to undesirable situations that are penalized by the third term in (5). This objective functions is constrained by a set of equality constraints (6) one per station $\mathrm{i}$ and per period $\mathrm{k}$. Each of them relates the volume $v_{i k}$ with the volume of reservoir $\mathrm{i}$ in period $\mathrm{k}-1$, with the inflow, with the outflow volume, with the water spill and with the water balance in the reservoirs upstream reservoir i and directly connected with it. Constraints (7) establish minimum and maximum limits for the liquid output volume of reservoir $i$, modeling for instance minimum flow requirements related with agriculture uses or ecological reasons. Constraints (8) bound the hourly volumes of reservoir $i$ and constraints (9) and (10) bound the generation and pumping water flows of station $i$ in period k. Finally, constraints (11) indicate that the water spill is non-negative and (12) sets the volume of reservoir $i$ at the end of the planning period, that is, at the end of hour K.

\section{SOLUTION ALGORITHM AND IMPLEMENTED APPROACHES}

The optimization problem described in Section III was solved using Genetic Algorithms. GA's are population based approaches that aim at identifying good solutions to optimization problems emulating the natural principles of evolution. In brief, after coding a possible solution to the problem typically as a string of bits, it is created an initial population including a number of individuals or chromosomes. Then, each potential solution to the problem is evaluated using a fitness function that characterizes the goodness of each one. The population is then reproduced using the genetic operators of selection, crossover and mutation in order to create a new population. This new population is evaluated and if convergence was not yet achieved, then the algorithm iterates again.

The decision variables of the complete problem formulated in Section III include the discharge volume $q_{\text {Tik }}$, and the pumping volume, $q_{P i k}$, in each hour of the planning horizon. The literature on short term hydro scheduling suggests that current market mechanisms are inducing generation companies to generate or pump with water flows as large as possible namely to profit from selling electricity when prices are higher and to pay for pumping as little as possible when prices are low. The same conclusion is for instance reported in [13]. In this publication the generation and pumping volumes are decision variables but the result of the optimization procedure allocates these volumes at the maximum possible values in order to maximize the profit. If the volumes are taken at the maximum possible values either when generating or pumping, then these volumes are no longer decision variables of the problem and the profit maximization problem now aims at deciding the status of each station, that is, identifying the best location for the generation and pumping periods. Under this assumption, the GA implemented to solve this problem used the following coding for the status of each hydro station:

- $00-$ not active;

- 01 - operating as a pump;

- 10 - operating as a generator;

- 11 - not active.

If the hydro system includes two stations and the planning horizon has 24 hours, then an individual is coded by a string of 96 bits resulting of the multiplication of 24 periods by 2 stations by 2 bits per station. After setting the number of individuals in the population, the algorithm evolves according the following steps:

i) Initialization - the string of bits representing each individual is initialized using a random sampling procedure;

ii) Evaluation - each individual or chromosome corresponds to a possible solution to the problem and the quality of this solution is measured by the value of the objective function (5). To calculate this value, and for each station $\mathrm{i}$ in period $\mathrm{k}$ it is necessary to compute the output hydro power in case it is operating as a generator or the pumping demand power in case it is in state 01 . This is done using expressions (2) or (4) in function of $q_{i k}$ and of the head $h_{i k}$. Recall that $q_{i k}$ is set at $q_{q} T_{i}^{\max }$ for state 10 and at $q P_{i}^{\max }$ for state 01 . Using the generation or pumping powers and a specified set of electricity prices it is possible to compute (5) for each individual along the planning period. It is still necessary to check if the solution associated to each individual belongs to the set of feasible solutions, that is, if it does not violate any constraint of the problem. Using the $q_{i k}$ values of each station, one computes the hourly volumes of station i $v_{i k}$ using (6) and compares it with the limits given by (7) and (8). If the volume in period $\mathrm{k}$ exceeds the maximum value then the variable $s_{i k}$ becomes non zero in order to balance equation (6) again. If the volume $v_{i k}$ is smaller than the minimum limit then the value of the optimization function (5) is penalized using a negative term that is proportional to square of the difference between the value of $v_{i k}$ and the violated limit. A similar penalty term is used if the final volume $v_{i k}$ for $\mathrm{k}=\mathrm{K}$ is different of the preestablished value given by constraint (12);

iii) Convergence check - after evaluating the population, the convergence criteria is checked. We considered that the algorithm converged if the average value of the fitness function of all individuals in the population did not change more than a specified percentage if compared with the results obtained for a number of previous iterations, if the standard deviation of the fitness function of all individuals is small enough and if the largest value of the fitness function (the one of the most adapted individual) did not change more than a specified percentage for a number of iterations;

iv) Genetic operators - if the convergence check was 
not successful, then the algorithm proceeds using the selection, the crossover and the mutation operators to create a new population. In the first place, pairs of individuals compete in a stochastic tournament to select the most adapted ones. Then, the crossover operator acts over some selected individuals and finally the mutation operator is used to introduce diversity in the population. At the end of this procedure, a new population is formed and the algorithm gets back to the evaluation step.

The previous algorithm represents a general approach that can now be adapted in order to increase the complexity and the realism of the model. The three designed approaches are as follows:

i) Model 1 - in the first place, we designed a very simplified version of the problem assuming that the head is constant and that the head loss term is neglected in (2) and (4). This model also assumes that the generation or pumping flows are at their maximum values and that they are equal. In real cases, these maximum flows will be reduced due to head variations. However, admitting that the head remains unchanged originates that the maximum generation and pumping flows also remain unchanged;

ii) Model 2 - in this model, we continue admitting that generation of pumping flows are at the maximum but these maximum values are not constant and equal from one iteration to the next one. Under this approach, at the end of each iteration the volumes $v_{i k}$ given by (6) are used to update the head $h_{i k}$ and then this is used to update the maximum flows using (14) and (15) as detailed in [15].

$$
\begin{aligned}
& q T_{i}^{\max }=q T n_{i} \cdot \sqrt{\frac{h_{i k}}{h T n_{i}}} \\
& q P_{i}^{\max }=q P n_{i}-\delta_{i} \cdot\left(h_{i k}-h P n_{i}\right)
\end{aligned}
$$

In these expressions and for station i, $q T n_{i}$ is nominal turbine discharge volume, $q P n_{i}$ is the nominal pumping volume, $h T n_{i}$ is the nominal turbine head, $h P n_{i}$ is the nominal pumping head and $\delta_{i}$ is the pumping coefficient. These are parameters characteristic of each reservoir and correspond to inputs to the problem. It is clear that if the head is taken as constant and equal to nominal value, then the maximum flows also coincide with the nominal values either for generation or pumping. This would correspond to the simplification used in Model 1. Finally, in this approach we also considered the head loss term in (2) and (4) computed using the values obtained for $q_{i k}$ in the previous iteration;

iii) Model 3 - the final model incorporates all the enhancements of Model 2 and it also considers the impact of the hydro schedule on the electricity prices. It is clear that the presence of selling bids submitted to the electricity market at low bid prices tend to shift the aggregate selling curve to the right thus reducing the market price. If the station is pumping, then the aggregated demand increases thus originating an increase of the market price. In order to incorporate this impact, we admitted that the generation agent that is conducting this scheduling exercise has estimates for the hourly demand in the system and for the aggregated selling curve for all the remaining stations, except the ones being scheduled. Then at each iteration of the AG and for each of the individuals and for each hour $\mathrm{k}$, the demand of hour $\mathrm{k}$ is increased using the pumping powers of the stations in status 01 and reduced by the generated powers of the stations that are in status 10. This modified demand is used together with the aggregated selling curve of the remaining stations to update the electricity market price in hour $\mathrm{k}$. These modified prices are finally used together with the hydro schedule associated to each individual to compute the remuneration of the stations being planned as the difference between the amounts to receive and to pay. This procedure admits that the hydro stations being scheduled will bid at low price if they are at status 10 or will bid at a very large price if they are at status 01 to ensure that are their selling/buying bids are always accepted in each hour of the planning period.

\section{CASE STUDIES}

\section{A. Hydro system 1}

The hydro system 1 includes four reservoirs that are uncoupled from an hydro point of view and that have the characteristics detailed in Table I.

\section{TABLE I}

CHARACTERISTICS OF THE FOUR HYDRO STATIONS.

\begin{tabular}{|l|r|r|r|r|}
\hline & \multicolumn{1}{|c|}{ Res. 1 } & Res. 2 & \multicolumn{1}{|c|}{ Res. 3 } & Res 4 \\
\hline Initial volume $\left(\mathrm{hm}^{3}\right)$ & 900 & 20 & 30 & 9 \\
\hline Final volume $\left(\mathrm{hm}^{3}\right)$ & 900 & 20 & 30 & 9 \\
\hline Minimum volume $\left(\mathrm{hm}^{3}\right)$ & 800 & 10 & 10 & 8 \\
\hline Maximum volume $\left(\mathrm{hm}^{3}\right)$ & 1000 & 30 & 50 & 10 \\
\hline$q T_{i}^{\text {max }}\left(\mathrm{m}^{3} / \mathrm{s}\right)$ & 400 & 120 & 70 & 50 \\
\hline$q P_{i}^{\text {max }}\left(\mathrm{m}^{3} / \mathrm{s}\right)$ & 400 & 120 & 70 & 50 \\
\hline Minimum spill & 0 & 0 & 0 & 0 \\
\hline Head $(\mathrm{m})$ & 50 & 100 & 150 & 500 \\
\hline Discharge efficiency, $\mu_{T}$ & 0,88 & 0,89 & 0,89 & 0,90 \\
\hline Pumping efficiency, $\mu_{P}$ & 0,92 & 0,93 & 0,93 & 0,93 \\
\hline Inflow (m $/ \mathrm{s})$ & 0 & 0 & 0 & 0 \\
\hline
\end{tabular}

In the first place, we used Model 1 to plan the operation for a 48 hour period. Table II presents the hourly prices for the first 24 hours and then this set of prices repeated on the second set of 24 hours. Given that for each station the inflows are zero, the initial and final volumes are equal and the maximum discharge and pumping flows are also equal, it is immediate to conclude that the number of periods during which each station is operating as a generator has to be equal to the number of periods during which it is 
pumping. Therefore, using Model 1 and the data in Table I leads to a very simplified situation that was used to set a number of parameters of the GA for this system. As a result of these initial tests, we selected populations with 40 individuals, a rate of 0,8 for stochastic selection tournament and a mutation rate of 0,1 . Using these values, Figs. 3 to 6 present the generation/pumping periods for the four stations and the electricity price evolution.

TABLE II

ELECTRICITY PRICES IN €/MWh FOR A 24 HOUR PERIOD.

\begin{tabular}{|c|c|c|c|c|c|}
\hline Hour & Price & Hour & Price & Hour & Price \\
\hline 1 & 55,00 & 9 & 56,16 & 17 & 58,50 \\
\hline 2 & 45,00 & 10 & 61,75 & 18 & 62,00 \\
\hline 3 & 46,50 & 11 & 72,50 & 19 & 90,60 \\
\hline 4 & 45,44 & 12 & 71,74 & 20 & 85,00 \\
\hline 5 & 45,52 & 13 & 72,42 & 21 & 85,00 \\
\hline 6 & 45,51 & 14 & 66,25 & 22 & 80,47 \\
\hline 7 & 45,58 & 15 & 61,07 & 23 & 70,00 \\
\hline 8 & 55,49 & 16 & 58,95 & 24 & 79,72 \\
\hline
\end{tabular}

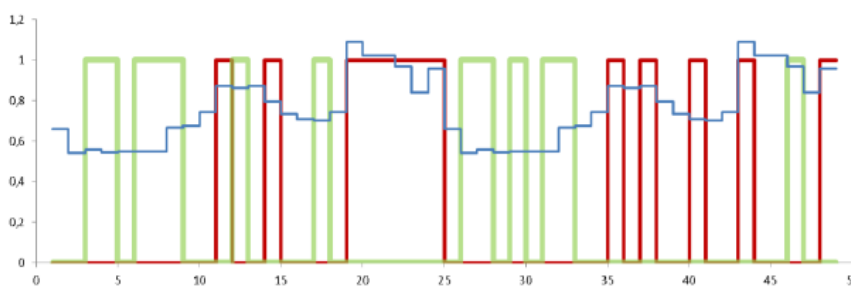

Fig. 3 - Generation and pumping periods for station 1 (generation - red line, pumping - green line, price - blue line in $€ / \mathrm{MWh}$ ).

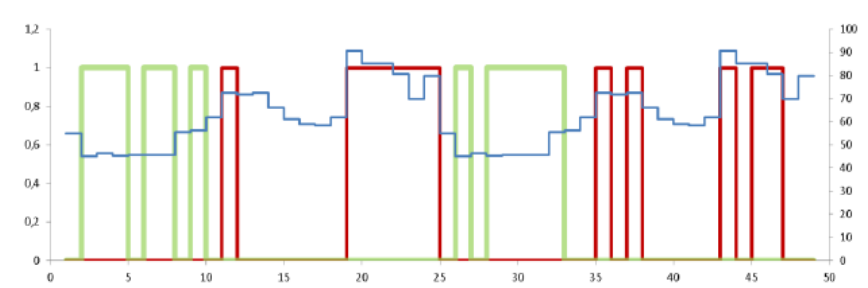

Fig. 4 - Generation and pumping periods for station 2 (generation - red line, pumping - green line, price - blue line in $€ / M W h)$.

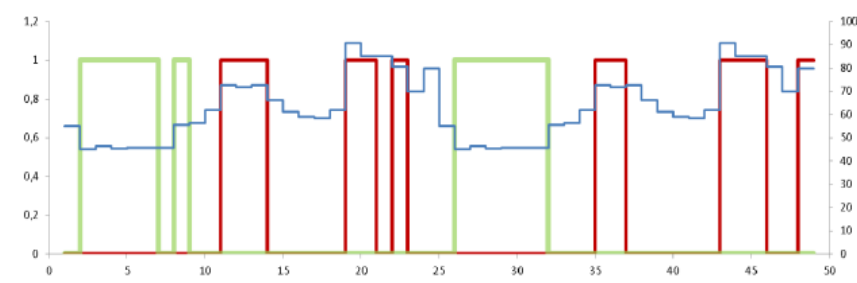

Fig. 5 - Generation and pumping periods for station 3 (generation - red line, pumping - green line, price - blue line in $€ / M W h$ ).

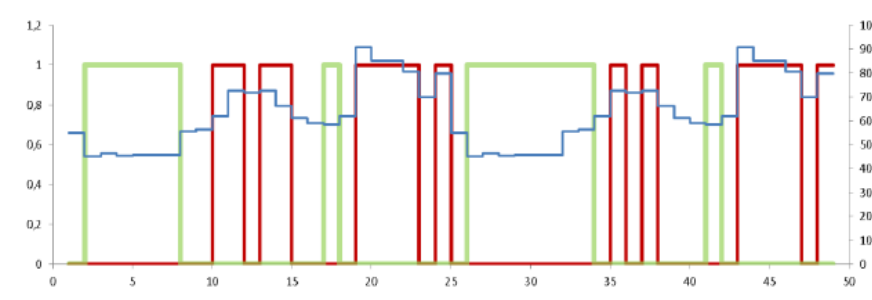

Fig. 6 - Generation and pumping periods for station 4 (generation - red line, pumping - green line, price - blue line in $€ / \mathrm{MWh}$ ).

These results confirm that generation periods tend to be located when the market price is larger and that pumping is done when the price is more reduced.

In a second step, we used Model 2 considering the inflows and the nominal discharge and pumping flows indicated in Table III. In each iteration, these values are used to update the maximum flow of each station using (14) and (15). Figs. 7 and 8 present the generation and pumping periods of stations 1 and 2 as well as the price evolution for a 24 hour period. In this case, the number of discharge and pumping periods are no longer equal because inflows are considered and the discharge and pumping volumes are not equal. Looking at Fig. 8, pumping is now limited to 2 periods since there are inflows all through the day as indicated in Table III.

TABLE III

CHARACTERISTICS OF THE RESERVOIRS FOR MODEL 2.

\begin{tabular}{|l|c|c|c|c|}
\hline & Res. 1 & Res. 2 & Res. 3 & Res. 4 \\
\hline Inflow hour 1 & 100 & 50 & 0 & 0 \\
\hline Inflow hour 2 & 100 & 50 & 0 & 0 \\
\hline Inflow hour 3 & 100 & 50 & 0 & 0 \\
\hline Inflow hour 4 & 100 & 50 & 0 & 0 \\
\hline Inflow hour 5 & 0 & 50 & 0 & 0 \\
\hline Inflow hour 6 & 0 & 50 & 0 & 0 \\
\hline Inflow hour 7 & 0 & 50 & 0 & 0 \\
\hline Inflow hour 8 & 0 & 50 & 0 & 0 \\
\hline Inflow hour 9 & 0 & 50 & 0 & 0 \\
\hline Inflow hour 10 & 0 & 50 & 0 & 0 \\
\hline Inflow hour 11 & 0 & 50 & 0 & 0 \\
\hline Inflow hour 12 & 0 & 50 & 0 & 0 \\
\hline Inflow hour 13 & 0 & 50 & 0 & 0 \\
\hline Inflow hour 14 & 0 & 50 & 100 & 0 \\
\hline Inflow hour 15 & 0 & 50 & 100 & 0 \\
\hline Inflow hour 16 & 0 & 50 & 100 & 0 \\
\hline Inflow hour 17 & 0 & 50 & 100 & 0 \\
\hline Inflow hour 18 & 0 & 50 & 100 & 0 \\
\hline Inflow hour 19 & 0 & 50 & 0 & 0 \\
\hline Inflow hour 20 & 0 & 50 & 0 & 0 \\
\hline Inflow hour 21 & 0 & 50 & 0 & 0 \\
\hline Inflow hour 22 & 0 & 50 & 0 & 0 \\
\hline Inflow hour 23 & 0 & 50 & 0 & 0 \\
\hline Inflow hour 24 & 0 & 50 & 0 & 0 \\
\hline$q T n_{i}$ (m)/s) & 400 & 120 & 70 & 50 \\
\hline$q P n_{i}$ (m) $\left./ \mathbf{s}\right)$ & 324 & 100 & 58 & 42 \\
\hline
\end{tabular}

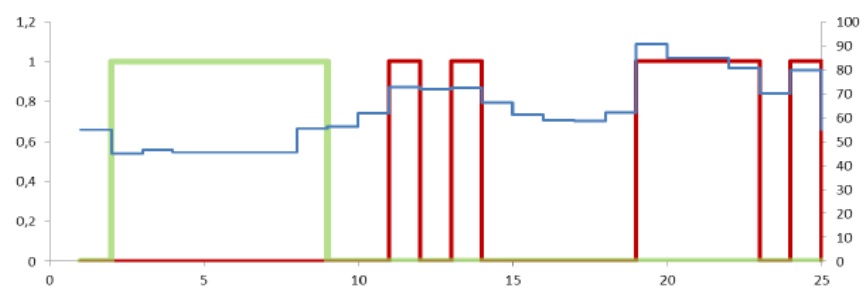

Fig. 7 - Generation and pumping periods for station 1 (generation - red line, pumping - green line, price - blue line in $€ / \mathrm{MWh}$ ).

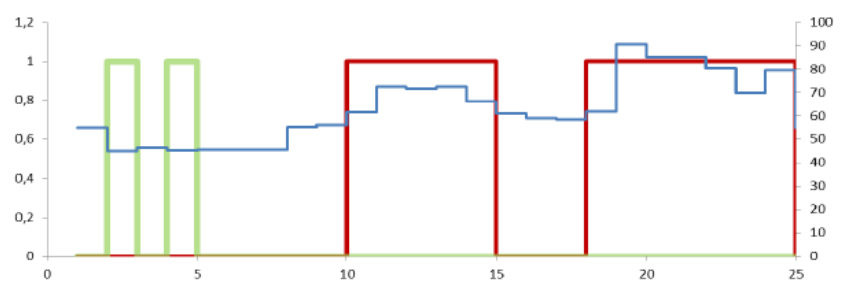

Fig. 8 - Generation and pumping periods for station 2 (generation - red line, pumping - green line, price - blue line in $€ / \mathrm{MWh}$ ).

Finally, Model 3 was used to incorporate the impact on the prices of the schedules obtained along the GA. For this purpose, we admitted that the demand to be supplied by the entire power system in which these four stations are 
included has the values indicated in Table IV for each hour of the planning day and that the estimated bid curve for the remaining stations in the power system is depicted in Fig. 9. Figs. 10 and 11 present the generation and pumping periods for stations 1 and 2 .

TABLE IV

DEMAND (MW) FOR A 24 HOUR PERIOD.

\begin{tabular}{|c|c|c|c|c|c|}
\hline Hour & $\begin{array}{c}\text { Demand } \\
(\mathrm{MW})\end{array}$ & Hour & $\begin{array}{c}\text { Demand } \\
(\mathrm{MW})\end{array}$ & Hour & $\begin{array}{c}\text { Demand } \\
(\mathrm{MW})\end{array}$ \\
\hline 1 & 3877,02 & 9 & 3992,15 & 17 & 4215,70 \\
\hline 2 & 2766,72 & 10 & 4506,86 & 18 & 4528,33 \\
\hline 3 & 2946,68 & 11 & 5308,86 & 19 & 6093,60 \\
\hline 4 & 2820,00 & 12 & 5260,33 & 20 & 5927,28 \\
\hline 5 & 2829,64 & 13 & 5303,81 & 21 & 5927,28 \\
\hline 6 & 2828,43 & 14 & 4872,77 & 22 & 5742,46 \\
\hline 7 & 2836,86 & 15 & 4447,81 & 23 & 5144,52 \\
\hline 8 & 3926,00 & 16 & 4257,36 & 24 & 4707,54 \\
\hline
\end{tabular}

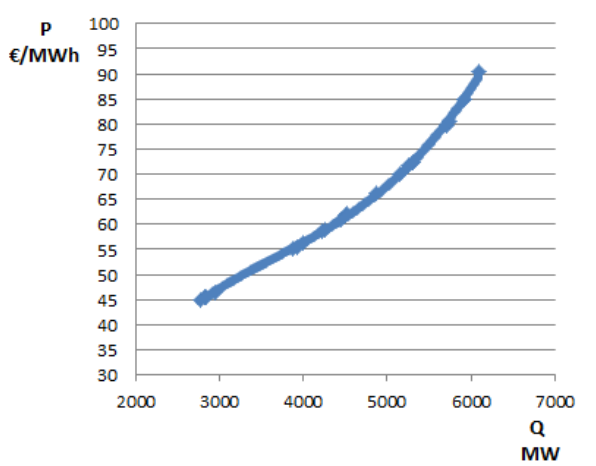

Fig. 9 - Approximate aggregated selling curve for the remaining generation system.

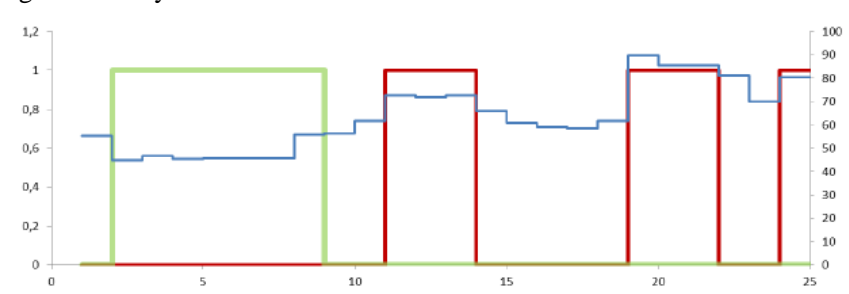

Fig. 10 - Generation and pumping periods for station 1 (generation - red line, pumping - green line, price - blue line in $€ / \mathrm{MWh}$ ).

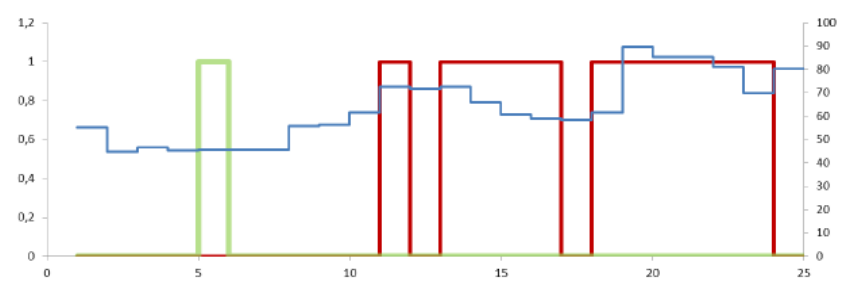

Fig. 11 - Generation and pumping periods for station 2 (generation - red line, pumping - green line, price - blue line in $€ / \mathrm{MWh}$ ).

As a result of this more accurate model, the electricity price in each hour changes along the iterative process typically increasing in the pumping periods regarding the initially input values because the demand increases. Conversely, the prices tend to decrease in the generation periods due to the presence of low price bids from the scheduled hydro stations. This ultimately reduces the operation profit that the generation company can obtain at the end of the day when compared with the value of the profit yield by Models 1 or 2 . For station 1, as an example, the profit was estimated to get reduced by $10,7 \%$ when comparing the results obtained with Models 1 and 3.

\section{B. Hydro system 2}

The second tested hydro system is the cascade of 8 stations in Fig. 12. To a certain extent, this cascade mirrors a Portuguese hydro basin and the main characteristics of the stations are listed in Table V. Among these stations, only $\mathrm{C}$ and $\mathrm{G}$ have pumping capacity. The inflows of hydro power plants $\mathrm{F}, \mathrm{G}$ and $\mathrm{H}$ are typically large, while the inflows of the remaining hydro stations are more reduced.

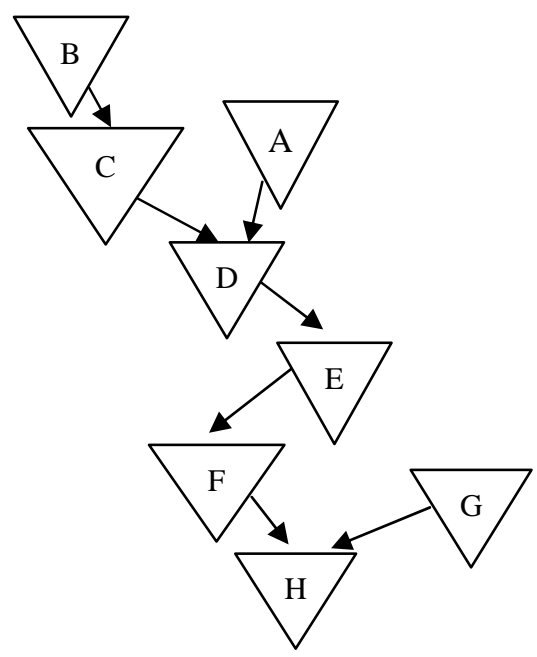

Fig. 12 - Cascade including 8 hydro stations.

TABLE V

CHARACTERISTICS OF THE 8 HYDRO STATIONS.

\begin{tabular}{|c|c|c|c|c|c|}
\hline Reservoir & $\begin{array}{c}\text { Vol. max } \\
\left(\mathrm{hm}^{3}\right)\end{array}$ & $\begin{array}{c}\text { Vol. min } \\
\left(\mathrm{hm}^{3}\right)\end{array}$ & $\begin{array}{c}q T n_{i} \\
\left(\mathrm{~m}^{3} / \mathrm{s}\right)\end{array}$ & $\begin{array}{c}q P n_{i} \\
\left(\mathrm{~m}^{3} / \mathrm{s}\right)\end{array}$ & $\begin{array}{c}h T n_{i} \\
(\mathrm{~m})\end{array}$ \\
\hline A & 31 & 25 & 120 & -- & 30 \\
\hline B & 83 & 71 & 500 & -- & 2 \\
\hline C & 216 & 156 & 210 & 162 & 116 \\
\hline D & 97 & 85 & 550 & -- & 30 \\
\hline E & 95 & 82 & 744 & -- & 27 \\
\hline F & 148 & 132 & 705 & -- & 33 \\
\hline G & 106 & 84 & 320 & 251 & 50 \\
\hline H & 110 & 94 & 750 & -- & 11 \\
\hline
\end{tabular}

Model 2 described in Section IV was used to plan the operation of this cascade along a week. This implicitly means that these stations are price-takers since we are neglecting the impact of the hydro schedules on the electricity prices. Fig. 13 presents the results that were obtained using Model 2 for the generation periods of the 8 stations and Fig. 14 indicates the pumping periods that were obtained for stations $\mathrm{C}$ and $\mathrm{G}$.

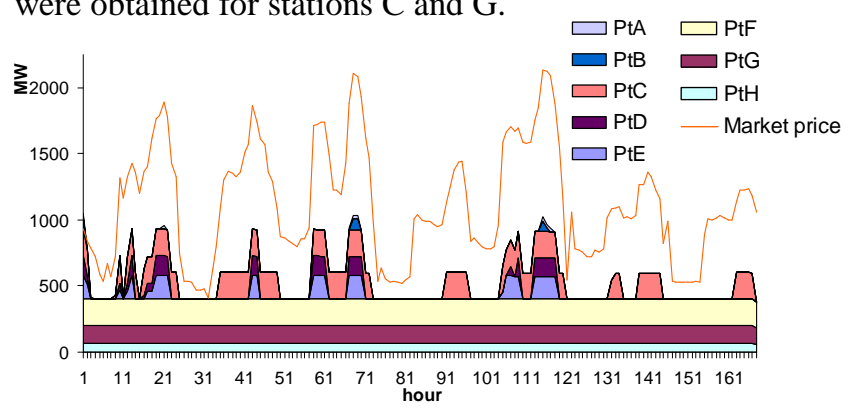

Fig. 13 - Generation of the 8 stations and evolution of the electricity price along the week. 


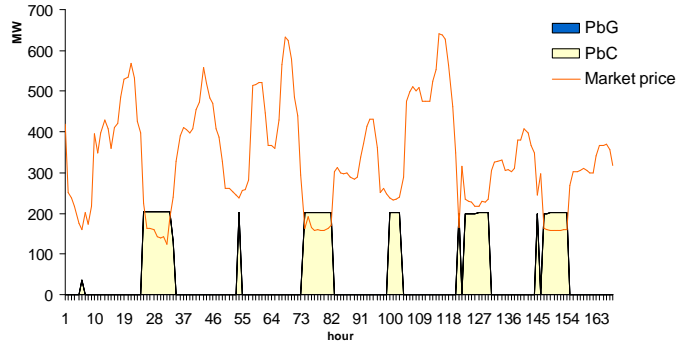

Fig. 14 - Pumping periods for stations $\mathrm{C}$ and $\mathrm{G}$ and evolution of the electricity market price along the week.

Regarding these results, it becomes clear that stations $\mathrm{F}$ and $\mathrm{G}$ are run of river and so they are scheduled to operate all over the week on the basis of the load diagram. On the other hand, station $\mathrm{G}$, although having pumping capacity, has large inflows and so it is also scheduled with a constant power output. The remaining stations are typically scheduled to operate when electricity prices are larger. As indicated before, station $G$ has large inflows during the whole week and so it has no pumping consumption. Differently, the hydro station $\mathrm{C}$ has more reduced inflows and so its pumping consumption is large. Finally, Fig. 14 confirms that pumping is used in the periods in which the electricity market price is more reduced. These results are well in line with the experience taken from the operation of the real cascade mentioned in the beginning of this section.

\section{CONCLUSIONS}

This paper detailed a model to develop short term operation planning studies for hydro systems to maximize the profit obtained by the generation agents along the planning period. The associated non-linear optimization problem was solved using Genetic Algorithms that proved to provide good results for all analysed cases, namely results in line with what was expected from real life experience. The obtained results confirm that under the current electricity market paradigm when an hydro station is operating it is typically running at its maximum generation or pumping flow and that generation periods tend to be located when the electricity prices are larger and pumping when they are more reduced. This allowed us to simplify the coding scheme of the GA solutions and confirmed that the maximization of short term profits is now the main driver for generation companies. The solution of one day or one week planning problem requires the input of the initial and final volumes of each station and in this work we admitted these values were provided by a medium term planning exercise. This means that the developed model can be used to help generation agents to prepare the bids to submit to the electricity market on a daily basis but has to interact with an upper level model to define the policy regarding the use of water. This is not addressed in this paper and it corresponds to a research topic to be followed in the future.

\section{REFERENCES}

[1] S.-C. Chang, C. H. Chen, I. K. Fong, P. B. Luh, "Hydroelectric Generation Scheduling With An Effective Differential Dynamic Programming Algorithm", IEEE Transactions on Power Systems, vol. 5, no. 3, pp. 737-743, August 1990.

[2] A. I. Cohen, V. R. Sherkat, "Optimization-Based Methods for Operations Scheduling", Proceedings of the IEEE, vol. 75, no. 12, pp. 1574-1591, 1987.
[3] L. Martinez, S. Soares, "Primal and Dual Stochastic Dynamic Programming in Long Term Hydrothermal Scheduling", in Proc. IEEE/PES T\&D Conf: Asia and Pacific, vol. 3, pp. 1283-1288, October 2004

[4] G. W. Wang, C. T. Su, "A Practical Mixed Integer Linear Programming Based Short Term Hydro Scheduling", in Proc. IEEE/PES T\&D Conf: Asia and Pacific, vol. 3, pp. 1606-1610, October 2002

[5] J. P. Catalão, S. J. P. S. Mariano, V. M. F. Mendes, and L. A. F. M. Ferreira, "Parameterisation effect on the behaviour of a headdependent hydro chain using a nonlinear model", Electrical Power Systems Research, vol. 76, no. 6/7, pp. 404-412, April 2006.

[6] K. P. Wong, Y. W. Yong, "A Parallel Simulated Annealing Algorithm for Short-Term Hydro Scheduling", in Proc. of the Second International Forum on Applications of Neural Networks to Power Systems Proceedings, pp. 335-340, Yokohama, Japan, 1993.

[7] R. H. Liang and Y. Y. Hsu, "Short-Term Hydro-Scheduling using Hopfield Neural Network", IEE Proc. G., T. \& D., vol. 143, no. 3, pp. 269-275, May 1996.

[8] R. H. Liang, Y. Y. Hsu, "Scheduling of hydroelectric generations using artificial neural networks", IEE Proc. G. T. \& D., vol. 141, no. 5, pp. 452-458, Sept. 1994.

[9] A. Mantawy, S. Soliman, M. El-Hawary, "A New Tabu Search Algorithm for the Long-Term Hydro Scheduling Problem", in Proc. of LESCOPE'2002, June 2002.

[10] J. García-González, E. Parrilla, J. Barquín, J. Alonso, A. SàizChicharro, A. González, "Under-relaxed Iterative Procedure for Feasible Short-Term Scheduling of a Hydro Chain", in Proc. IEEE Bologna Power Tech, vol. 2, June 2003.

[11] J. García-González, E. Parrilla, A. Mateo, "Profit-based optimal scheduling of hydro chains in the day-ahead electricity market", IIT internal report, Madrid, Spain, December 2004.

[12] J. García-González, E. Parrilla, A. Mateo, R. Moraga, "Building optimal generation bids of a hydro chain in the day-ahead electricity market under price uncertainty", in Proc. 9th PMAPS, Stockholm, Sweden, June 2006.

[13] J. V. Sousa, V. T. Mendes, J. T. Saraiva, "Estimation of the Remuneration of Hydro Plants in a Market Environment Using an Iterative Under-Relaxation Approach", in Proc. of the IEEE Bucharest Power Tech, IEEE BPT 2009, Bucharest, June 2009.

[14] G. S. Sampaio, "Optimization of the Operation of Hydro Stations Using Genetic Algorithms in Market Environment", MSc Thesis, Fac. de Engenharia da Universidade do Porto, FEUP, July 2012.

[15] VALORAGUA "A Model for the Optimal HydroThermal Management”, Internal Report, EDP Produção, June 1987.

\section{BIOGRAPHIES}

Gil Sampaio was born in Amarante, Portugal in 1987. In July 2012 he got his MSc from Faculdade de Engenharia da Universidade do Porto, FEUP and he is now working as junior consultant with Everis Portugal.

João Tomé Saraiva was born in Porto, Portugal in 1962. In 1987, 1993 and 2002 he got his MSc, PhD, and Agregado degrees in Electrical and Computer Engineering from FEUP, where he is currently Professor. In 1985 he joined INESC Porto where he is head researcher and collaborated in several projects namely in the scope of consultancy contracts wit the Portuguese Electricity Regulatory Agency.

José Carlos Sousa was born in Porto, Portugal in 1982. In 2005 and 2007 he got the licenciate and the MSc degrees from the Faculdade de Engenharia da Universidade do Porto, FEUP. Since 2006 he works in EDP Produção and currently he also is a PhD student at FEUP.

Virgílio Torrado Mendes was born in Porto, Portugal in 1959. In 1978 got the Bachelor of Mechanics Engineering from Instituto Superior de Engenharia do Porto and in 1981 he graduated in Mechanical Engineering from FEUP. In 1985 he joined EDP and since 1989 he works in expansion studies of power generation systems. He acted as invited expert by IEA for training courses in Mexico and Egypt.

\section{ACKNOWLEDGMENT}

This work is financed by the ERDF - European Regional Development Fund through the COMPETE Programme (operational programme for competitiveness) and by National Funds through the FCT - Fundação para a Ciência e a Tecnologia (Portuguese Foundation for Science and Technology) within project «FCOMP-01-0124-FEDER-037281» 